\title{
A varying-coefficient cox model for the effect of CA19-9 kinetics on overall survival in patients with advanced pancreatic cancer
}

\author{
Yuntao Chen ${ }^{1,2, *}$, Zhenyi Shao ${ }^{2, *}$, Wen Chen ${ }^{2}$, Hua Xie ${ }^{2}$, Zhenyu Wu ${ }^{1,2}$, Guoyou Qin ${ }^{1,2}$, \\ Naiqing Zhao ${ }^{1,2}$ \\ ${ }^{1}$ Department of Biostatistics, School of Public Health and Key Laboratory of Public Health Safety, Fudan University, Shanghai \\ 200032, China \\ ${ }^{2}$ Collaborative Innovation Center of Social Risks Governance in Health, Fudan University, Shanghai 200032, China \\ *Co-first authors \\ Correspondence to: Guoyou Qin, email: gyqin@fudan.edu.cn \\ Naiqing Zhao, email: nqzhao@shmu.edu.cn \\ Keywords: CA 19-9, pancreatic cancer, varying coefficient model, biomarker, prognosis \\ Abbreviations: PC: pancreatic cancer; OS: overall survival; CA19-9: carbohydrate antigen 19-9; PH: proportional hazard; HR: \\ hazard ratio. \\ Received: August 16, $2016 \quad$ Accepted: January 23, $2017 \quad$ Published: February 21, 2017 \\ Copyright: Chen et al. This is an open-access article distributed under the terms of the Creative Commons Attribution License \\ (CC-BY), which permits unrestricted use, distribution, and reproduction in any medium, provided the original author and source \\ are credited.
}

\section{ABSTRACT}

Purpose: To evaluate the relationship between serum CA19-9 and overall survival in patients with advanced pancreatic cancer.

Methods: 109 advanced pancreatic cancer patients with gemcitabine based firstline chemotherapy were included. The effect of pretreatment CA19-9 level on overall survival was modeled by Cox proportional hazard regression. The effect of CA19-9 kinetics on overall survival was modeled by an extended Cox regression with a time varying coefficient and a time varying covariate.

Results: Univariate analysis indicated that baseline CA19-9 correlated with os $(H R=1.66, p<0.01)$ and this association remained significant within multivariate analysis (HR $=1.56, P<0.01)$. For the analysis of CA19-9 kinetics, the extended Cox model showed that the effect of CA19-9 on overall survival changed with time: increased in the first two months and reached the top at a HR of about 2, then decreased for the next two months to a HR of about 1.56 and finally tended to be stable. The combination of pretreatment CA19-9 and CA19-9 at 2 month may better evaluate the patients' prognosis compared to pretreatment CA19-9 alone.

Conclusion: Pretreatment CA19-9 and CA19-9 kinetics may serve as a useful serum biomarker in advanced pancreatic cancer.

\section{INTRODUCTION}

Pancreatic cancer (PC) is associated with a very poor prognosis, highlighted by the close parallel between disease incidence and mortality [1]. More than $80 \%$ of patients are initially diagnosed in an advanced stage of disease, where the potential curative resection is no longer possible. Gemcitabine is still being regarded as the standard chemotherapy for the treatment of locally advanced and metastatic disease [2]. However, treatment effects remain moderate with median overall survival (OS) times in the range of 5 to 8 months and 1-year survival rates in the range of $17-25 \%$ [3]. Therefore, in addition to a good therapeutic option, establishing clinically relevant prognostic biomarkers is also very important for this aggressive disease.

More than 2000 studies of biomarkers in pancreatic cancer has been published, implicating more than 2000 different genes and proteins [4]. Many studies concerning the correlation of SMAD4 genetic and molecular alteration with prognosis has been conducted. PC patients with SMAD4 expression had significantly longer survival as compared to those lacking SMAD4 expression [5]. Circulating tumor cells and circulating tumor DNA constitute easily accessible 
blood-borne tumor biomarkers that may prove their clinical interest for screening, early diagnosis and metastatic risk assessment of PC [6]. However, none of these biomarkers have been showed to possess the requisite sensitivity/ specificity to be introduced in clinical use. The carbohydrate antigen 19-9 (CA19-9) is still the most commonly used and best validated serum tumor marker for pancreatic cancer diagnosis in symptomatic patients and for monitoring therapy in patients with pancreatic adenocarcinoma [7].

CA19-9 was first discovered in 1979 by researchers using monoclonal antibodies to isolate tumor associated antigens in colorectal carcinoma and two years later was also found to be produced by pancreatic carcinoma $[8,9]$. However, approximately $5 \%$ to $14 \%$ of the population is Lewis antigen $\mathrm{A}$ and $\mathrm{B}$ negative $\left(\mathrm{Le}^{\mathrm{a}-\mathrm{b}-}\right)$, and is also considered CA19-9 nonsecretory (CA19-9 < 5U/mL), which is correlated with poor survival [10]. Therefore these patients are excluded from this study. Since accurate determination of treatment response by imaging often remains difficult ( e.g., due to the desmoplastic stroma reaction induced by the tumor itself in surrounding soft tissue ), the serum tumor marker CA19-9 has been studied for several years if it could serve as an appropriate surrogate parameter of treatment efficacy [11].

Several previous studies suggested a significant correlation between serum CA19-9 and survival end point in patients receiving systemic chemotherapy for advanced pancreatic cancer [12-19]. Most of these studies discussed the relation between the pretreatment CA19-9 and OS $[12,13]$. Some studies still considered a constant effect of CA19-9 on OS though a CA19-9 kinetics analysis was taken. [18-21] It was reported that the value of individual prognostic factors may change dependent on the length of the follow-up time; for example, the effect of a treatment can be strong immediately after treatment but fades with time [22]. Using Cox proportional hazards $(\mathrm{PH})$ regression when the PH assumption was violated may produce biased results [23]. Time dependency has been accounted for and reported in oncology publications, such as in breast, colon and gastric cancer studies [24-28]. But it has not been considered in a pancreatic cancer study before. In addition, these published studies were limited to fixed covariates, measured at time of diagnosis. In this study, an extended cox model with a time varying coefficient and a time varying covariate was used to solve the problem that the effect of CA19-9 on OS was changing and a serial CA19-9 measurements should be included in the model.

The aims of this retrospective study were as follows: first, to evaluate the prognostic role of pretreatment CA199 in patients with advanced pancreatic cancer. Second, to construct an extended cox model which can consider not only the dynamic change of CA19-9 during the treatment, but also the different effect of CA19-9 on OS in the course of the treatment. Last, according to the above knowledge about the entire path variation of CA19-9's effect on OS during chemotherapy, to better predict the PC patients' prognosis in combination with the pretreatment CA19-9.

\section{RESULTS}

\section{Patient characteristics}

Basic characteristics of 109 PC patients included in this study were listed in Table 1 . The mean of age before chemotherapy for all patients was 64 years, ranging from 39 to 86 years. Males and females were almost equivalent in number ( 59 vs 50). Tumors were more likely to occur at the head or neck of pancreas $(n=50,46 \%)$ than the body or tail $(n=35,32 \%)$. The tumor location of the remaining 24 cases couldn't be figured out due to the insufficient diagnostic information (diagnosis such as pancreatic carcinoma in situ or pancreatic malignant tumor). The majority of patients had metastasis before the chemotherapy $(n=70,64 \%)$, and $71 \%$ of which metastasized to liver with the remaining spreading to lung, bone, brain and so on. The median OS of the patients was 7.4 months (range, 1 - 34.1 months). The pretreatment CA19-9 measurements were done in the previous one month before the start of first-line chemotherapy. The median pretreatment CA19-9 in the study population was $532.5 \mathrm{U} /$ $\mathrm{mL}$ (range, 5.1 - $10000 \mathrm{U} / \mathrm{mL}$ ). 77\% (84) of patients had 2 or more CA19-9 measurements during the chemotherapy (median, 3; range, 1-16). The median CA19-9 level during the chemotherapy was $823.9 \mathrm{U} / \mathrm{mL}$ (range, 5.3 - $12777 \mathrm{U} / \mathrm{mL}$ ).

\section{Pretreatment CA19-9}

The pretreatment CA19-9 level was identified as a prognostic factor for OS in univariate and multivariate analyses, respectively. Every 9-fold CA19-9 increase means a HR of $1.66(95 \% C I: 1.30-2.12, P<0.01)$ in the univariate Cox regression model, and after adjusting for age, sex and chemotherapy (Gemcitabine combined with other drugs vs Gemcitabine alone), the HR for the effect of every 9-fold CA19-9 increase was estimated with 1.56 (95\% CI: $1.20-2.01, P<0.01)$. Residual analyses showed adequate fit of the multivariate Cox model especially with regard to the proportional hazards assumption and linearity assumption.

\section{Changeable effect with CA19-9 kinetics}

Considering the varying effect of CA19-9 on OS, we firstly confirmed this effect by a schoenfeld residual plot. (Supplementary Figure 1) Though the test for PH assumption was not significant ( $p=0.78$ ), we thought it should be rejected for that a quadratic shape for $\beta(t)$ might be apparent on the plot, but be undetected by the test for linear slope [29]. In order to get a more objective and comprehensive result, CA19-9 kinetics was applied in place of the CA19-9 measurement at one time point. Finally a Cox model with a time varying coefficient and a time varying covariate was constructed and the result showed that the effect of CA19-9 on OS changed with time: increased in the first two months and reached the top at a HR of about 2, then decreased for the next two months to a HR of about 1.56 and finally tended to be stable. (Figure 1). 
Table 1: Baseline patient characteristics $(n=109)$

\begin{tabular}{|c|c|c|}
\hline & No. & $\%$ \\
\hline \multicolumn{3}{|l|}{ Gender } \\
\hline Male & 59 & 54.13 \\
\hline Female & 50 & 45.87 \\
\hline \multicolumn{3}{|l|}{ Chemotherapy } \\
\hline Gemcitabine alone & 41 & 37.61 \\
\hline Gemcitabine combined with other drugs* & 68 & 62.39 \\
\hline \multicolumn{3}{|l|}{ Primary pancreas tumor } \\
\hline Head & 50 & 45.87 \\
\hline Body or tail & 35 & 32.11 \\
\hline Unknown & 24 & 22.02 \\
\hline \multicolumn{3}{|l|}{ Stage of disease } \\
\hline Locally advanced & 10 & 9.17 \\
\hline Metastatic & 70 & 64.22 \\
\hline Unknown & 29 & 26.61 \\
\hline \multicolumn{3}{|l|}{ Distant metastasis } \\
\hline Liver & 50 & 71.43 \\
\hline Lung & 2 & 2.86 \\
\hline bone & 4 & 5.71 \\
\hline Abdomen & 5 & 7.14 \\
\hline
\end{tabular}

*other drugs include oxaliplatin, erlotinib, capecitabine, or 5-Fluorouracil. Most of the combination are gemcitabine and oxaliplatin.

\section{The combination of pretreatment CA19-9 and CA19-9 at two months better predicts PC patients' prognosis}

In order to achieve a unique definition of (individualized and stage-adapted) cutoff points that will help to separate different prognostic subgroups, we divided the patients at different time points into two groups $(\geq 1000 \mathrm{U} / \mathrm{mL}$ and $<1000 \mathrm{U} / \mathrm{mL})$ according to the CA19-9 level [30]. We chose $1000 \mathrm{U} / \mathrm{mL}$ for two reasons. One was that previous studies took it [31], and the other was that many CA19-9 levels far larger than $1000 \mathrm{U} /$ $\mathrm{mL}$ had been identified as $1000 \mathrm{U} / \mathrm{mL}$ in this database. Those patients with CA19-9 $<1000 \mathrm{U} / \mathrm{mL}$ had a longer median survival compared to those with CA19-9 levels $\geq 1000 \mathrm{U} / \mathrm{mL}$ at $1,2,3$ months (9 vs 5.9 months, $p<$ 0.01 ; 9.8 vs 5.9 months, $p<0.01$; 10.6 vs 7.1 months, $p=0.01)$ using log-rank test while the results were not significant at 4, 5, 6 months (7.5 vs 7.8 months, $p=0.82$; 9.4 vs 7.9 months, $p=0.64$; 9.6 vs 10.2 months, $p=0.65$ ). (Figure 2) After adjusting for age, pretreatment CA19-9, sex and chemotherapy, the HRs for the effect of CA199 ( $\geq 1000 v s<1000 \mathrm{U} / \mathrm{mL})$ on OS showed a tendency similar to Figure 1. Because of the limited sample size and adjustment of pretreatment CA19-9 in the model, we did not get a statistically significant result. HR reached the top at 2 month though the $p$ value was not significant. (Table 2) Therefore, CA19-9 at 2 month might be the most influential prognostic factor in the course of chemotherapy. So the combination of pretreatment CA19-9 and 2 month CA19-9 was used to evaluate the patients' prognosis, and those patients with pretreatment CA19-9 $<1000 \mathrm{U} / \mathrm{mL}$ and 2 month CA19-9 $<1000 \mathrm{U} / \mathrm{mL}$ had a longer median survival compared to those with both $\geq 1000 \mathrm{U} / \mathrm{mL}$ (8.8 vs 5.9 months, $p<0.01$ ). (Figure 3) If pretreatment CA19-9 was considered alone, patients with pretreatment CA19-9 $<1000 \mathrm{U} / \mathrm{mL}$ had a longer median survival compared to those with pretreatment CA19-9 $\geq 1000 \mathrm{U} / \mathrm{mL}$ (8.0 vs 5.9 months, $p=0.02$ ). Above results suggest that adding a 2 month CA19-9 do predict the prognosis of the subgroup with pretreatment CA19-9 $<1000 \mathrm{U} / \mathrm{mL}$ more accurately though there is no difference in the prediction of the subgroup with pretreatment CA19-9 $\geq 1000 \mathrm{U} / \mathrm{mL}$. Due to the fact that different clinical studies reported a wide discrepancy for a CA19-9 cutoff level (200-1212 U/ml) that makes identifying prognostic relevant subgroups possible [11], we also performed a sensitivity analysis based on different CA19-9 cutoff levels to check the robustness of the study results. As we can learn from 


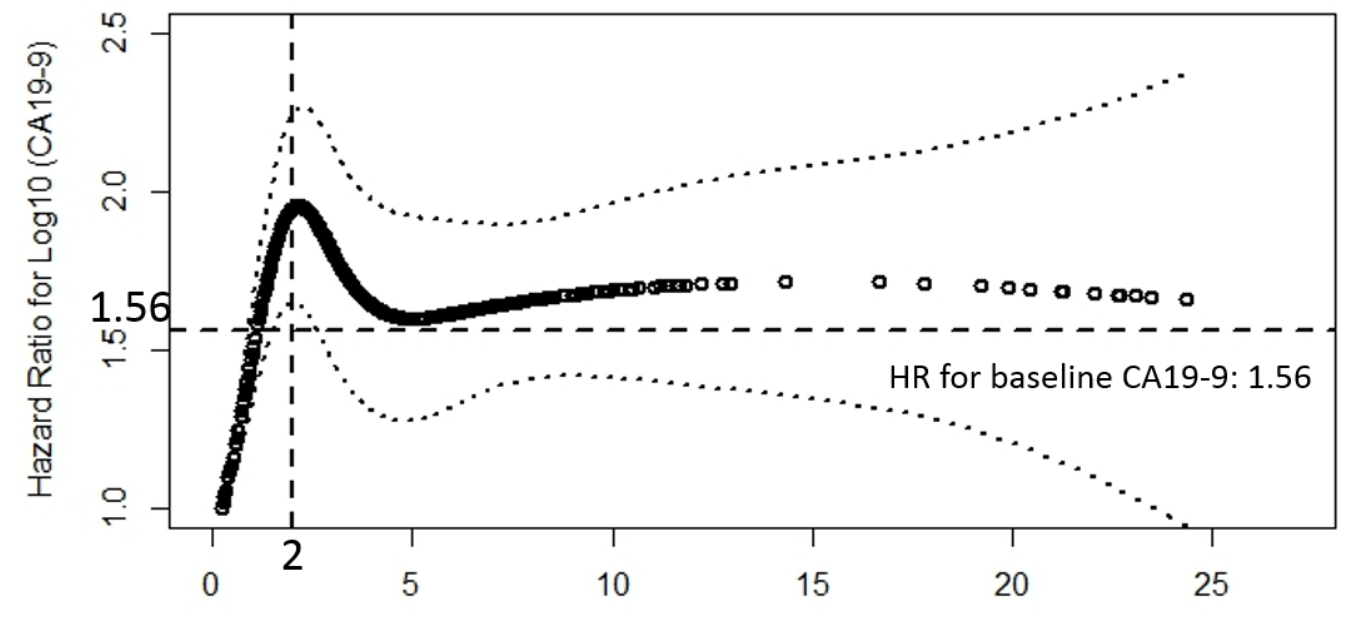

Follow-up (month)

Figure 1: Estimates of the effect of peri-treatment CA19-9 on overall survival using the natural spline, presented as hazard ratio (solid line) and $\mathbf{9 5 \%}$ CI (dashed lines) in extended Cox model with time-varying covariates and hazard ratio. The horizontal reference line was set at 1.56, which was the HR for the effect of baseline CA19-9 on OS. The vertical reference line was set at 2 month, when HR for the effect of peri-treatmnet CA19-9 on OS reached the top.

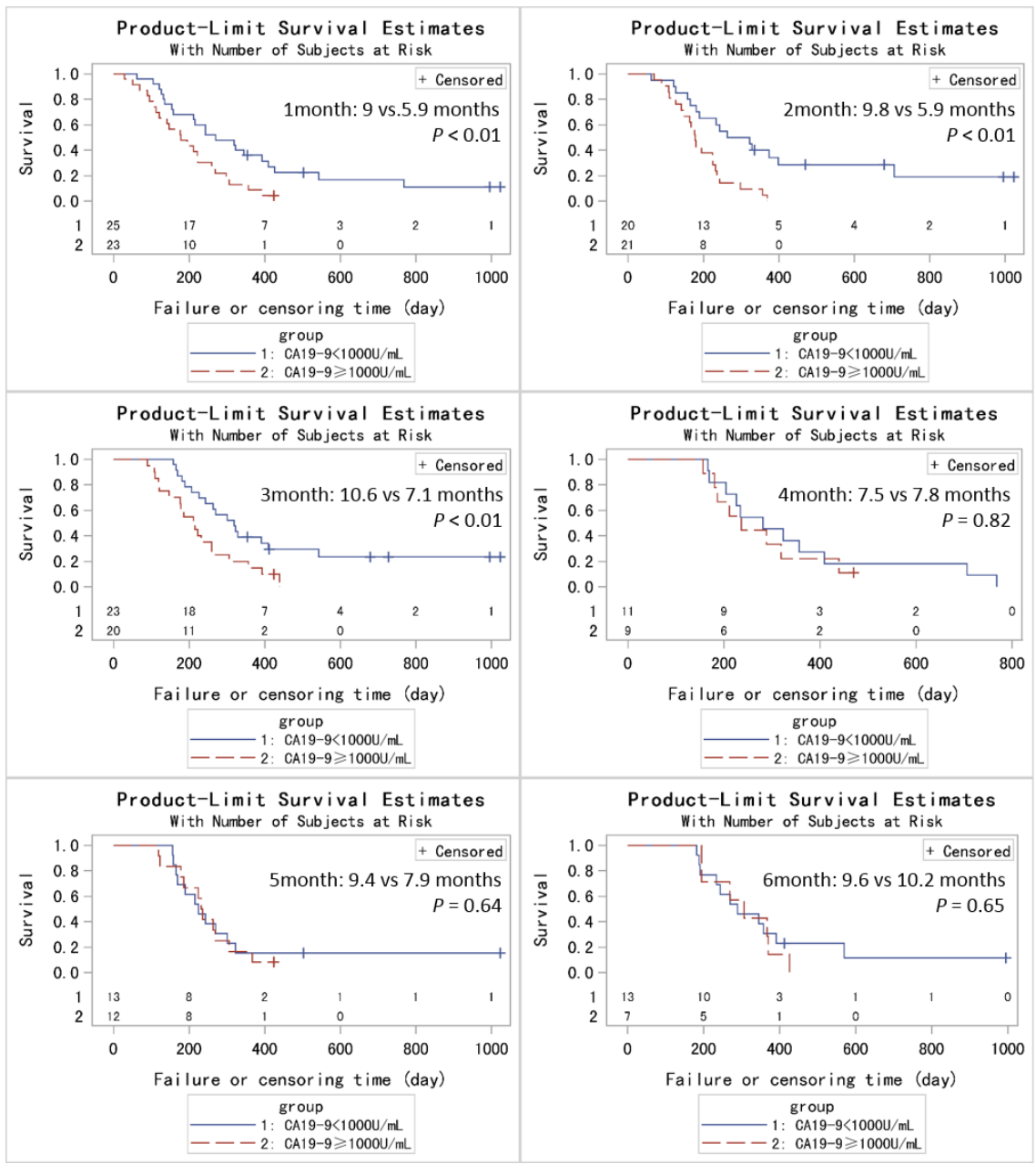

Figure 2: Kaplan-Metier analysis of OS for PC patients based on CA19-9 levels at different time points (1-6 months). 
Table 2: Impact of post-treatment CA19-9 levels $(\geq 1000 \mathrm{vs}<1000 \mathrm{U} / \mathrm{mL})$ on $\mathrm{OS}$ in a univariate and multivariate Cox model

\begin{tabular}{|c|c|c|c|c|c|}
\hline \multirow{2}{*}{ Time } & \multirow{2}{*}{$\mathbf{N}$} & \multicolumn{2}{|c|}{ Univariate Cox model } & \multicolumn{2}{|c|}{ Multivariate Cox model } \\
\hline & & Crude HR (95\% CI) & $\boldsymbol{P}$ & Adjusted HR* (95\% CI) & $P$ \\
\hline 1 month & 48 & $2.27(1.20-4.31)$ & 0.01 & $0.56(0.03-11.77)$ & 0.71 \\
\hline 2 month & 41 & $3.11(1.49-6.49)$ & $<0.01$ & $2.55(0.89-7.27)$ & 0.08 \\
\hline 3 month & 43 & $2.34(1.19-4.58)$ & 0.01 & $2.46(0.99-6.10)$ & 0.05 \\
\hline 4 month & 25 & $1.21(0.52-2.81)$ & 0.66 & $2.00(0.68-6.92)$ & 0.21 \\
\hline 5 month & 20 & $1.26(0.48-3.28)$ & 0.64 & $1.35(0.35-5.18)$ & 0.66 \\
\hline 6 month & 20 & $1.25(0.47-3.31)$ & 0.65 & $1.63(0.32-8.19)$ & 0.56 \\
\hline
\end{tabular}

* adjust for age, baseline CA19-9, sex and chemotherapy.

Table 3: Prognostic value of the combination of pretreatment CA19-9 and CA19-9 at 2 month compared to the pretreatment CA19-9 alone

\begin{tabular}{|c|c|c|}
\hline \multirow{2}{*}{ CA19-9 cutoff level, U/ml } & \multicolumn{2}{|c|}{ Median survival, months } \\
\hline & Pretreatment CA19-9a & Pretreatment CA19-9 and CA19-9 at 2 month $^{\mathrm{b}}$ \\
\hline 1000 & 8.0 vs. $5.9(p=0.0171)$ & 8.8 vs. $5.9(p=0.0043)$ \\
\hline 800 & 7.9 vs. $6.0(p=0.1141)$ & 8.8 vs. $6.0(p=0.0273)$ \\
\hline 600 & 8.1 vs. $5.9(p=0.0344)$ & 9.8 vs. $5.9(p=0.0114)$ \\
\hline 400 & 8.8 vs. $6.0(p=0.0138)$ & 10.8 vs. $6.0(p=0.0079)$ \\
\hline 200 & 10.8 vs. $6.0(p=0.0060)$ & 12.5 vs. $6.0(p=0.0022)$ \\
\hline
\end{tabular}

${ }^{a}$ categorized by pretreatment CA19-9 alone (pretreatment CA19-9 $<$ m vs. pretreatment CA19-9 $\geq$ m, m: CA19-9 cutoff level).

${ }^{\mathrm{b}}$ categorized by the combination of pretreatment CA19-9 and CA19-9 at 2 month (pretreatment CA19-9 $<$ m and CA19-9 at 2 month $<$ m vs. pretreatment CA19-9 $\geq$ m and CA19-9 at 2 month $\geq m$, m: CA19-9 cutoff level).

Table 3 and Figure 4, the choice of cutoff levels did not influence the main conclusion.

\section{DISCUSSION}

Detection of suitable predictive biomarkers to evaluate the treatment effect of chemotherapy and predict the prognosis is still a major challenge for advanced pancreatic cancer patients. CA19-9 is viewed as the most important serum biomarker in pancreatic cancer [32-34]. It greatly reflects tumor burden and activity more than any other marker that has been reported so far $[33,35$, 36]. Though there were many studies confirming the association between the pretreatment CA19-9 level and OS $[12,13]$ and some investigators reported the effect of an entire path of CA19-9 levels on OS as well [18], to our knowledge a Cox model considering both varying effect of CA19-9 on OS and CA19-9 kinetics as a continuous variable has not been constructed yet to probe into the comprehensive relationship between CA19-9 and OS.
In our study, the method of Therneau and Grambsch (2000) [29] was used to expand the data set for the time dependent covariate CA19-9. And this method allowed us to make use of every CA19-9 measurement of one single patient, compared to the use of the pretreatment CA19-9 only, which may get a more precise determination of its prognostic biological significance. Meanwhile, a varying effect of CA19-9 kinetics on OS was also considered to get a more comprehensive result. The method to explore the varying effect of a time dependent covariate on outcome has been previously used in other researches. Bin et al. used it to investigate the effect of age at a marker event on age at menopause [37]. Robert et al. also took this method to explore the varying effect of race and maternal smoking on the risk of mortality post-LMP (last menstrual period) [38]. However, the previous studies all discussed a categorical and unordered time dependent variable, and to our knowledge, the present study is the first one to study the varying effect of an ordered time dependent variable. 
Our study finally met its predefined end point: pretreatment CA19-9 level was found to be an independent prognostic factor for OS in univariate and multivariate analyses. The effect of CA19-9 levels on OS varied in the different course of the treatment, and more specifically the association between CA19-9 and OS became stronger with time for the first two months, and then weaker with time for the next two months, finally tended to be stable. Meanwhile the effect of the pretreatment CA19-9 level on OS was smaller than that of the post-treatment from the first to the fifth month. (Figure 1) Though at least 6 months are recommended for patients to receive

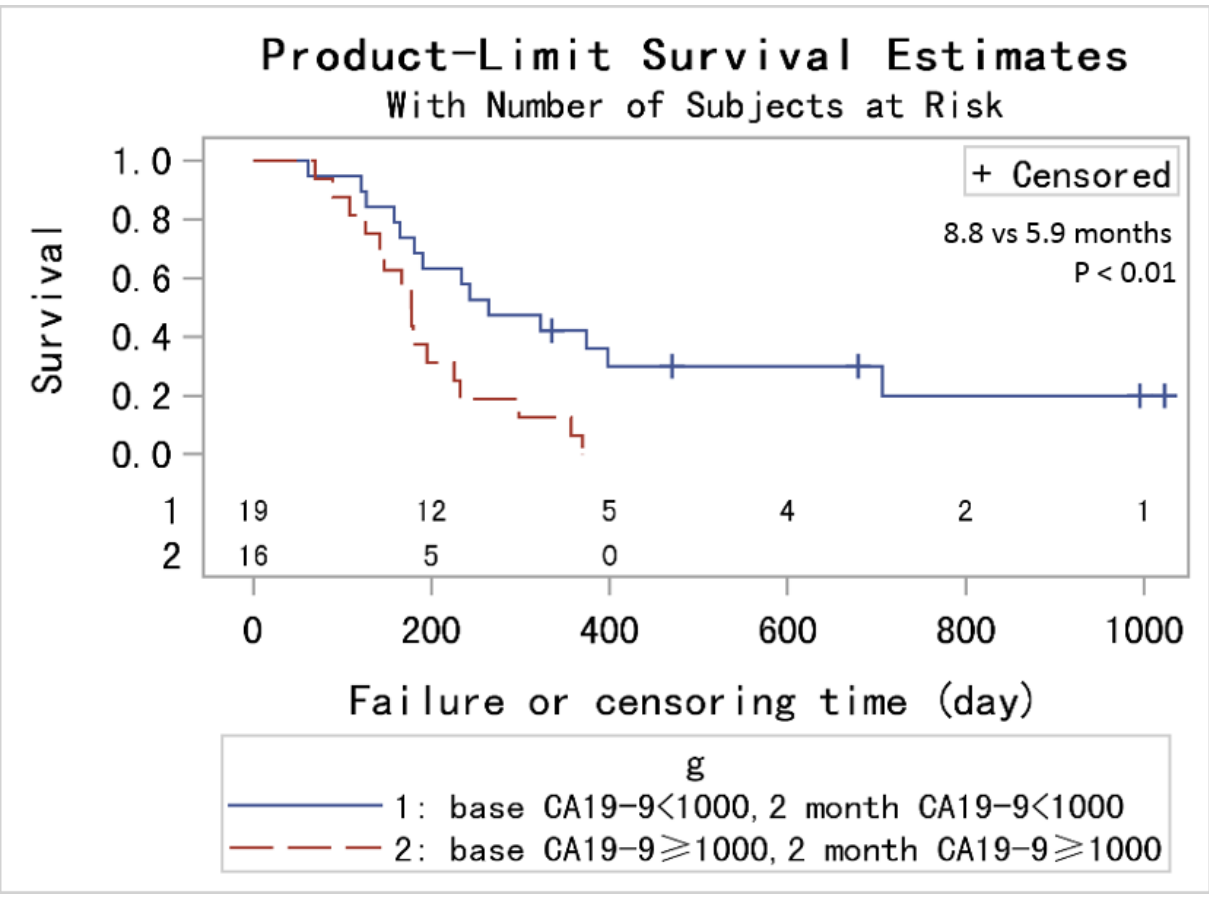

Figure 3: Kaplan-Metier analysis of OS for PC patients with pretreatment CA19-9 $<1000$ U/mL and 2 month CA19-9 $<1000 \mathrm{U} / \mathrm{mL}$ compared to those with both $\geq 1000 \mathrm{U} / \mathrm{mL}$.

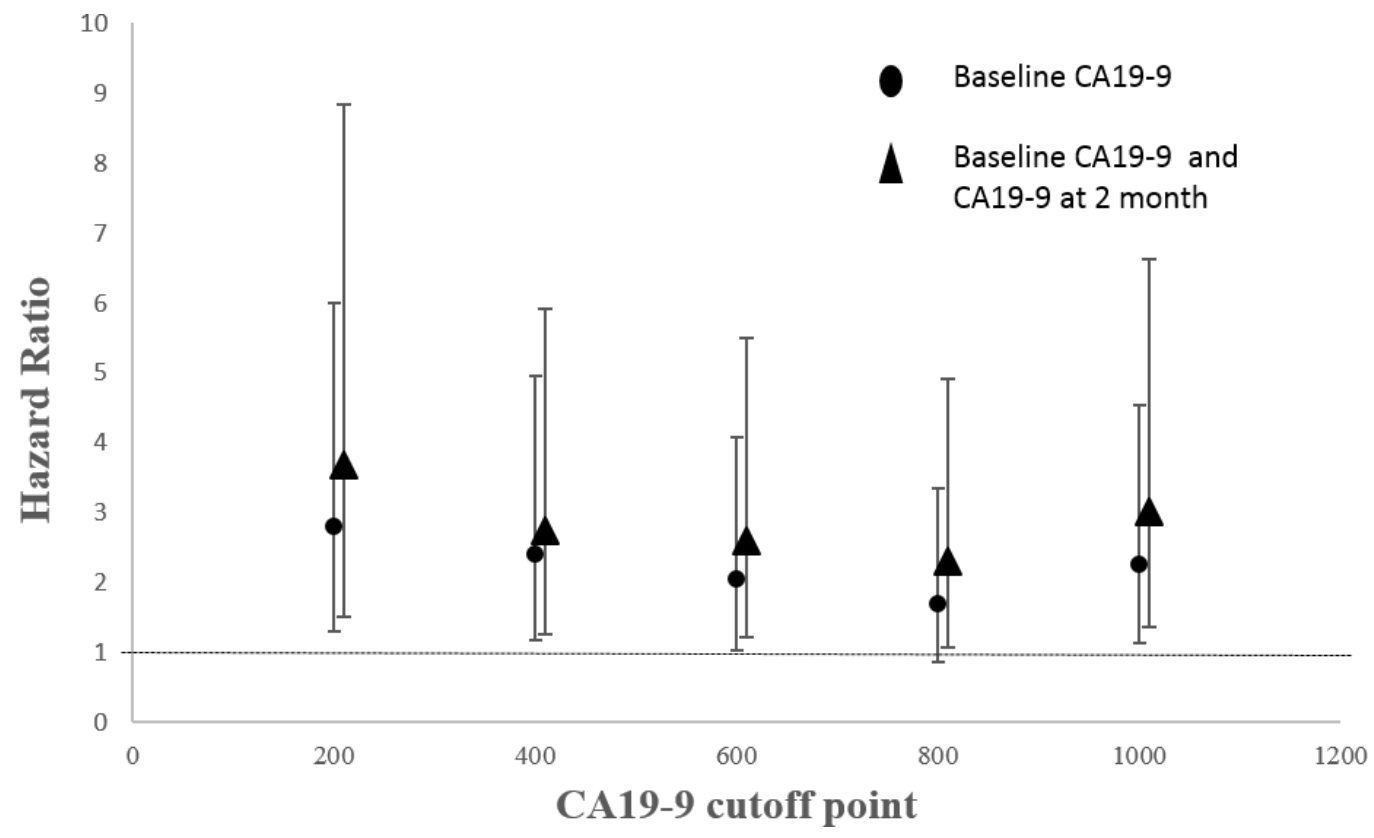

Figure 4: HRs with 95\% CIs by different CA19-9 cutoff levels. 
chemotherapy, there are no RCT data to support duration of initial treatment. In practice, total duration of firstline chemotherapy is variable and dependent on patient tolerability and tumor response [39]. What's more, with current chemotherapy regimens, the median survival for patients with unresectable tumors is 9-11 months (this study: 7.4 months) [40]. Therefore, we can infer that patients usually receive first-line chemotherapy not longer than 6 months. One might conclude that adding a CA19-9 during the chemotherapy can better predict the prognosis compared to a pretreatment CA19-9 alone. The key problem is that there is no evidence about how long after the initial of treatment we should take the CA19-9 measurement though most of the studies consider 2 month as that time point $[19,41]$. In this study we found the time point ' 2 month' was exactly the time when the increase of the CA19-9 level showed the strongest mortality risk. This was confirmed by the sequent analysis which showed that patients with CA19-9 $\geq 1000 \mathrm{U} / \mathrm{mL}$ had a worse prognosis in the follow-up of 2 and 3 month after adjusting other covariates. The HR for the effect of CA199 on OS reached the top at 2 month, and then weakened. (Table 2) And we found that PC patients with pretreatment CA19-9 $<1000 \mathrm{U} / \mathrm{mL}$ and 2 month CA19-9 $<1000 \mathrm{U} / \mathrm{mL}$ had a longer median survival compared to those with both $\geq 1000 \mathrm{U} / \mathrm{mL}$ (8.8 vs 5.9 months, $p<0.01)$. Sensitivity analysis based on different cutoff levels showed consistent results. An interesting thing was that the change of the cutoff level didn't affect the survival (median survival: 6 months) of the subgroup with pretreatment CA19-9 $\geq$ $1000 \mathrm{U} / \mathrm{mL}$ whether a 2 month CA19-9 level was larger than $1000 \mathrm{U} / \mathrm{mL}$ or not. This might explain why we did not get a statistically significant result in multivariate analyses which adjusted the pretreatment CA19-9 in the model (Table 2). However, for the subgroup with pretreatment CA19-9 < $1000 \mathrm{U} / \mathrm{mL}$, patients with 2 month CA19-9 < $1000 \mathrm{U} / \mathrm{mL}$ had a longer median survival compared to those with 2 month CA19-9 $\geq 1000 \mathrm{U} / \mathrm{mL}$. One might conclude that patients with pretreatment CA19-9 $\geq 1000$ $\mathrm{U} / \mathrm{mL}$ unavoidably will have a poor prognosis even though they undergo systemic chemotherapy. Perhaps we should focus more on the patients with pretreatment CA19-9 < $1000 \mathrm{U} / \mathrm{mL}$ and take effective measures to maintain a stable CA19-9 level. We cannot discuss the subgroups (1: pretreatment CA19-9 $<1000 \mathrm{U} / \mathrm{mL}$, two months $\geq$ CA19$91000 \mathrm{U} / \mathrm{mL}$; 2: pretreatment CA19-9 $\geq 1000 \mathrm{U} / \mathrm{mL}$, two months CA19-9 $<1000 \mathrm{U} / \mathrm{mL}$ ) due to the limited sample size.

The main limitations of this investigation arise from its retrospective nature and the consequent missing data distributing unevenly in different variables. Indicators of PC progression could have been superior endpoints to illustrate the influence of pretreatment CA19-9 or CA19-9 kinetics on PC survival compared with OS. However, due to the unavailability of the PC progression information, the relationship between CA19-9 and PC progression could not be discussed. And likewise, some possible prognostic factor such as tumor location, tumor size and pathological characteristics had not be adjusted when performing multivariate analysis because of data constraints. However, since that the effect of CA19-9 on OS was our real concern rather than other possible covariates, it was reported that the Andersen-Gill model gives a nearly unbiased estimate of the treatment effect, even when an important covariate has been omitted. The naive estimate of variance may be too small, but the robust estimate corrects for this by using "sandwich" estimator [29]. Lastly the authors are aware of the fact that the direct clinical applicability of results generated from this novel analytic model may be limited. However, given the lack of researches consisting of both an entire path of CA199 monitoring and varying effect of CA19-9 in advanced pancreatic cancer, if validated by a prospective clinical trial in an independent patient cohort, our approach may in fact provide a further step for establishing this biomarker as a useful tool in pancreatic cancer.

In conclusion, this retrospective cohort study constructs an entire path effect variation of CA19-9 kinetics on OS by modeling an extended Cox regression with a time varying coefficient and a time varying covariate. Then the CA19-9 measurement at 2 month is confirmed to better predict the advanced PC patients' prognosis in combination with the pretreatment CA199 measurement. These results suggest that pretreatment CA19-9 and CA19-9 kinetics may serve as a useful serum biomarker to predict patients' prognosis in advanced pancreatic cancer. But the underlying mechanisms behind this relationship should be explored for possible therapeutic intervention measures.

\section{MATERIALS AND METHODS}

\section{Study design}

We performed a retrospective review in a mega population-based electronic inpatients database. This database was established in 2011 and had kept updating on daily basis ever since, all country-level and above hospitals within Shanghai Metropolitan area, China, which were also qualified for cancer diagnosis, were responsible for tracking and reporting relevant information of all admitted patients, such as specifics of diagnosis, results of tests and examination, and treatment details. 669 historically confirmed PC patients were identified with prior chemotherapy from this database between 1 January 2012 and 31 December 2013. PC patients who underwent a pancreatectomy were excluded $(N=152)$, and those without Gemcitabine in their first-line chemotherapy $(N=88)$ or without a pretreatment CA19-9 measurement $(N=269)$ or with a pretreatment CA19-9 measurement < $5 \mathrm{U} / \mathrm{mL}(N=18)$ or with a single pretreatment CA19-9 
measurement $(N=33)$ were also excluded from the study. Finally, we got 109 PC patients in our study.

The outcome of interest was the OS, which was defined as the time interval between the initiation of chemotherapy and death from any cause. The date of death for PC patients was determined through external matching with the death registration system. The matching deadline was set as 31 January 2015. The whole study was approved by Institutional Research Ethics Board of Fudan University.

\section{CA19-9 measurement}

Due to the fact that the patients were from different hospitals in Shanghai and used different instruments to measure serum CA19-9, method-related differences may exist in results of CA19-9, which was not ideal, given that CA19-9 measurements could vary according to the detection method [42]. However, all the CA19-9 measurements for any given patient were performed at the same laboratory, ensuring some degree of intrapatient consistency. To exclude patients with undetectable CA199 (Le ${ }^{\text {a-b- }}$ phenotype), pretreatment CA19-9 measurement $>5 \mathrm{U} / \mathrm{mL}$ was requested for each included patient. Pretreatment CA19-9 level was defined as the last measurement before the first use of chemotherapeutic drugs. For the analysis of CA19-9 kinetics, serial CA199 measurements were not taken on a defined schedule. The frequency of monitoring was directly related to how actively patients were surveyed and depended partly on whether they received maintenance chemotherapy. We retained the first one if there were several times of CA199 measurements in one week.

\section{Statistical analysis}

All CA19-9 measurements were treated as a continuous variable and were transformed by taking the logarithm $\left[\log _{10}(\mathrm{CA} 19-9)\right]$. The effect of pretreatment CA19-9 levels on OS was modeled by Cox proportional hazard regression and the strength of effect was measured by hazard ratio (HR). The association between CA19-9 kinetics and OS was also modeled by Cox model where CA19-9 measurements were transformed again by taking the logarithm $\left[\log _{10}\right.$ (CA19-9)]. Counting process approach was applied in Cox model to deal with time varying covariates[ $\left[\log _{10}\right.$ (CA19-9) kinetics], this approach splits every single original observation into a group of "subobservations" at time points when a specific covariate varied. It assumes that the value of this time-varying covariate stays put between two consecutive time points. Thus, within the transformed database, for every "subobservation," all covariates will be static, and multiple failuretime Cox model under different further assumptions, such as fixed or changed baseline hazard, constant or variant effect along with survival time, can therefore be applied. The major difference between multiple failuretime Cox proportional hazard model and common Cox proportional hazards model is that, the former further adjusts for correlation between "subobservations" stemmed from the same original observation to get a robust variance, by using "sandwich" estimator for instance [43]. Considering that the effect of CA199 on OS may change over time, the $\mathrm{PH}$ assumption was investigated graphically by a schoenfeld residual plot (Supplementary Figure 1) and the result indicated that the regression coefficient $\beta(\cdot)$ of a timedependent covariate is a function of the follow-up time. (Supplementary Figure 2) Then a Cox model with a varying-coefficient and a varying-covariate was considered, which could be written as,

$$
\lambda\left(t \mid \mu_{i}, X_{i}(t)\right)=\lambda_{0}(t) \exp \left[\gamma \cdot \mu_{i}+\beta(t) \cdot X_{i}(t)\right]
$$

where $\boldsymbol{\lambda}\left(\mathbf{t} \mid \boldsymbol{\mu}_{\mathrm{i}}, \mathbf{X}(\mathbf{t})\right)$ refers to the instantaneous hazard rate at time point $\mathbf{t}$ given $\boldsymbol{\mu}_{\mathrm{i}}$ and $\mathbf{X}(\mathbf{t}) ; \boldsymbol{\lambda}_{\mathbf{0}}(\mathbf{t})$ is the baseline hazard function, $\boldsymbol{\mu}_{\mathbf{i}}$ describes the baseline covariates of patient $\mathbf{i}$. $\mathbf{X}_{\mathrm{i}}(\mathbf{t})$ describes the onedimensional marker measurement of patient $\mathbf{i}$ at time t. The regression coefficients of the Cox regression for the baseline covariates are given by the vector $\gamma$, and the coefficient for the effect of the biomarker is quantified by $\boldsymbol{\beta}(\mathbf{t})$, which is a function of the followup time. The function $\boldsymbol{\beta}(\mathbf{t})$ is obtained by estimating $\boldsymbol{\beta}$ at numerous intervals in time and then smoothing over these pointwise estimates using natural cubic spline functions. OS was displayed using KaplanMeier survival curves and the differences between subgroups were compared using the log-rank test. We checked the assumptions of Cox regression models by using graphically based residual analyses where we evaluated the proportional hazards assumption, the functional form of the continuous covariates and possible influential observations. These analyses were performed with SAS (version 9.3, SAS Institute Inc., Cary, NC, ) and R (version 3.0.2), and a probability $P$ value of 0.05 or lower was considered statistically significant.

\section{Authors' contributions}

C.Y., Q.G. and Z.N. conceived of the idea, designed the study. S.Z., C.W. and X.H. collected and analyzed data. C.Y. and Q.G. confirmed statistical analyses. C.Y. and W.Z. interpreted data and wrote the manuscript. C.Y., S.Z. and Q.G. revised the manuscript.

\section{ACKNOWLEDGMENTS}

The authors thank the staffs of the database and death registration system for their efforts in data collection and preprocessing. 


\section{CONFLICTS OF INTEREST}

The authors declare that they have no conflicts of interest.

\section{GRANT SUPPORT}

This research was supported by National Natural Science Foundation of China (Grant 81273187), National Science and Technology Major Project of the Peoples Republic of China (Major Grant 2012ZX09303-013-014) and Key Lab of Health Technology Assessment, Ministry of Health of the Peoples Republic of China.

\section{REFERENCES}

1. Siegel R, Ma J, Zou Z, Jemal A. Cancer statistics, 2014. CA Cancer J Clin. 2014; 64:9-29.

2. Burris HR, Moore MJ, Andersen J, Green MR, Rothenberg ML, Modiano MR, Cripps MC, Portenoy RK, Storniolo AM, Tarassoff P, Nelson R, Dorr FA, Stephens CD, et al. Improvements in survival and clinical benefit with gemcitabine as first-line therapy for patients with advanced pancreas cancer: a randomized trial. J Clin Oncol. 1997; 15:2403-2413.

3. Heinemann V, Boeck S, Hinke A, Labianca R, Louvet C. Meta-analysis of randomized trials: evaluation of benefit from gemcitabine-based combination chemotherapy applied in advanced pancreatic cancer. Bmc Cancer. 2008; 8:82.

4. Harsha HC, Kandasamy K, Ranganathan P, Rani S, Ramabadran S, Gollapudi S, Balakrishnan L, Dwivedi SB, Telikicherla D, Selvan LD, Goel R, Mathivanan S, Marimuthu A, et al. A compendium of potential biomarkers of pancreatic cancer. Plos Med. 2009; 6:e1000046.

5. Xia X, Wu W, Huang C, Cen G, Jiang T, Cao J, Huang K, Qiu Z. SMAD4 and its role in pancreatic cancer. Tumour Biol. 2015; 36:111-119.

6. Riva F, Dronov OI, Khomenko DI, Huguet F, Louvet C, Mariani P, Stern MH, Lantz O, Proudhon C, Pierga JY, Bidard FC. Clinical applications of circulating tumor DNA and circulating tumor cells in pancreatic cancer. Mol Oncol. 2016; 10:481-493.

7. Scara S, Bottoni P, Scatena R. CA 19-9: Biochemical and Clinical Aspects. Adv Exp Med Biol. 2015; 867:247-260.

8. Koprowski H, Herlyn M, Steplewski Z, Sears HF. Specific antigen in serum of patients with colon carcinoma. Science. $1981 ; 212: 53-55$.

9. Koprowski H, Steplewski Z, Mitchell K, Herlyn M, Herlyn D, Fuhrer P. Colorectal carcinoma antigens detected by hybridoma antibodies. Somatic Cell Genet. 1979; 5:957-971.

10. Tempero MA, Uchida E, Takasaki H, Burnett DA, Steplewski Z, Pour PM. Relationship of carbohydrate antigen 19-9 and Lewis antigens in pancreatic cancer. Cancer Res. 1987; 47:5501-5503.

11. Boeck S, Stieber P, Holdenrieder S, Wilkowski R, Heinemann V. Prognostic and therapeutic significance of carbohydrate antigen 19-9 as tumor marker in patients with pancreatic cancer. Oncology-Basel. 2006; 70:255-264.

12. Saad ED, Machado MC, Wajsbrot D, Abramoff R, Hoff PM, Tabacof J, Katz A, Simon SD, Gansl RC. Pretreatment CA 19-9 level as a prognostic factor in patients with advanced pancreatic cancer treated with gemcitabine. Int $\mathrm{J}$ Gastrointest Cancer. 2002; 32:35-41.

13. Maisey NR, Norman AR, Hill A, Massey A, Oates J, Cunningham D. CA19-9 as a prognostic factor in inoperable pancreatic cancer: the implication for clinical trials. Br J Cancer. 2005; 93:740-743.

14. Lee J, Park JO, Kim WS, Lee SI, Song SY, Lim DH, Choi SH, Heo JS, Lee KT, Lee JK, Kim K, Jung CW, Im YH, et al. Phase II study of gemcitabine combined with uraciltegafur in metastatic pancreatic cancer. Oncology-Basel. 2004; 66:32-37.

15. Cantore M, Rabbi C, Fiorentini G, Oliani C, Zamagni D, Iacono C, Mambrini A, Del FA, Manni A. Combined irinotecan and oxaliplatin in patients with advanced pretreated pancreatic cancer. Oncology-Basel. 2004; 67:93-97.

16. Ishii H, Okada S, Sato T, Wakasugi H, Saisho H, Furuse J, Ishikawa O, Matsuno S, Yokoyama S. CA 19-9 in evaluating the response to chemotherapy in advanced pancreatic cancer. Hepatogastroenterology. 1997; 44:279-283.

17. Wasan HS, Springett GM, Chodkiewicz C, Wong R, Maurel J, Barone C, Rosbrook B, Ricart AD, Kim S, Spano JP. CA 19-9 as a biomarker in advanced pancreatic cancer patients randomised to gemcitabine plus axitinib or gemcitabine alone. Br J Cancer. 2009; 101:1162-1167.

18. Boeck S, Haas M, Laubender RP, Kullmann F, Klose C, Bruns CJ, Wilkowski R, Stieber P, Holdenrieder S, Buchner H, Mansmann U, Heinemann V. Application of a TimeVarying Covariate Model to the Analysis of CA 19-9 as Serum Biomarker in Patients with Advanced Pancreatic Cancer. Clin Cancer Res. 2010; 16:986-994.

19. Ziske C, Schlie C, Gorschluter M, Glasmacher A, Mey U, Strehl J, Sauerbruch T, Schmidt-Wolf IG. Prognostic value of CA 19-9 levels in patients with inoperable adenocarcinoma of the pancreas treated with gemcitabine. Br J Cancer. 2003; 89:1413-1417.

20. Klapdor R, Bahlo M, Babinski A, Klapdor S. CA19-9 serum concentrations--analysis of the serum kinetics during firstline therapy of pancreatic cancer in relation to overall survival. Anticancer Res. 2010; 30:1869-1874.

21. Boeck S, Schulz C, Stieber P, Holdenrieder S, Weckbach S, Heinemann V. Assessing prognosis in metastatic pancreatic cancer by the serum tumor marker CA 19-9: pretreatment levels or kinetics during chemotherapy? Onkologie. 2007; 30:39-42. 
22. Bellera CA, MacGrogan G, Debled M, de Lara CT, Brouste V, Mathoulin-Pelissier S. Variables with time-varying effects and the Cox model: some statistical concepts illustrated with a prognostic factor study in breast cancer. Bmc Med Res Methodol. 2010; 10:20.

23. O'Quigley J, Pessione F. The problem of a covariate-time qualitative interaction in a survival study. Biometrics. 1991; 47:101-115.

24. Hilsenbeck SG, Ravdin PM, de Moor CA, Chamness GC, Osborne CK, Clark GM. Time-dependence of hazard ratios for prognostic factors in primary breast cancer. Breast Cancer Res Treat. 1998; 52:227-237.

25. Quantin C, Abrahamowicz M, Moreau T, Bartlett G, MacKenzie T, Tazi MA, Lalonde L, Faivre J. Variation over time of the effects of prognostic factors in a populationbased study of colon cancer: comparison of statistical models. Am J Epidemiol. 1999; 150:1188-1200.

26. Yoshimoto M, Sakamoto G, Ohashi Y. Time dependency of the influence of prognostic factors on relapse in breast cancer. Cancer-Am Cancer Soc. 1993; 72:2993-3001.

27. Schmitt M, Thomssen C, Ulm K, Seiderer A, Harbeck N, Hofler H, Janicke F, Graeff H. Time-varying prognostic impact of tumour biological factors urokinase (uPA), PAI-1 and steroid hormone receptor status in primary breast cancer. Br J Cancer. 1997; 76:306-311.

28. Bolard P, Quantin C, Esteve J, Faivre J, Abrahamowicz M. Modelling time-dependent hazard ratios in relative survival: application to colon cancer. J Clin Epidemiol. 2001; 54:986-996.

29. Therneau TM, Grambsch PM. (2000). Modeling Survival Data: Extending the Cox Model. (New York: Spring-Verlag).

30. Boeck S, Stieber P, Holdenrieder S, Wilkowski R, Heinemann V. Prognostic and Therapeutic Significance of Carbohydrate Antigen 19-9 as Tumor Marker in Patients with Pancreatic Cancer. Oncology-Basel. 2006; 70:255-264.

31. Liu L, Xu H, Wang W, Wu C, Chen Y, Yang J, Cen P, Xu J, Liu C, Long J, Guha S, Fu D, Ni Q, et al. A preoperative serum signature of CEA + /CA125+/CA19-9 $\geq 1000 \mathrm{U} / \mathrm{mL}$ indicates poor outcome to pancreatectomy for pancreatic cancer. Int J Cancer. 2015; 136:2216-2227.

32. Ferrone CR, Finkelstein DM, Thayer SP, Muzikansky A, Fernandez-delCastillo C, Warshaw AL. Perioperative CA19-9 levels can predict stage and survival in patients with resectable pancreatic adenocarcinoma. J Clin Oncol. 2006; 24:2897-2902.
33. Poruk KE, Gay DZ, Brown K, Mulvihill JD, Boucher KM, Scaife CL, Firpo MA, Mulvihill SJ. The clinical utility of CA 19-9 in pancreatic adenocarcinoma: diagnostic and prognostic updates. Curr Mol Med. 2013; 13:340-351.

34. Balzano G, Di Carlo V. Is CA 19-9 useful in the management of pancreatic cancer? Lancet Oncol. 2008; 9:89-91.

35. Locker GY, Hamilton S, Harris J, Jessup JM, Kemeny N, Macdonald JS, Somerfield MR, Hayes DF, Bast RJ. ASCO 2006 update of recommendations for the use of tumor markers in gastrointestinal cancer. J Clin Oncol. 2006; 24:5313-5327.

36. Xu HX, Chen T, Wang WQ, Wu CT, Liu C, Long J, Xu J, Zhang YJ, Chen RH, Liu L, Yu XJ. Metabolic tumour burden assessed by (1)(8)F-FDG PET/CT associated with serum CA19-9 predicts pancreatic cancer outcome after resection. Eur J Nucl Med Mol Imaging. 2014; 41:1093-1102.

37. Nan B, Lin X, Lisabeth LD, Harlow SD. A varyingcoefficient Cox model for the effect of age at a marker event on age at menopause. Biometrics. 2005; 61:576-583.

38. Platt RW. A Proportional Hazards Model with Timedependent Covariates and Time-varying Effects for Analysis of Fetal and Infant Death. Am J Epidemiol. 2004; 160:199-206.

39. Balaban EP, Mangu PB, Khorana AA, Shah MA, Mukherjee $\mathrm{S}$, Crane $\mathrm{CH}$, Javle MM, Eads JR, Allen P, Ko AH, Engebretson A, Herman JM, Strickler JH, et al. Locally Advanced, Unresectable Pancreatic Cancer: American Society of Clinical Oncology Clinical Practice Guideline. J Clin Oncol. 2016; 34:2654-2668.

40. Akinleye A, Iragavarapu C, Furqan M, Cang S, Liu D. Novel agents for advanced pancreatic cancer. Oncotarget. 2015; 6:39521-39537. doi: 10.18632/oncotarget.3999.

41. Halm U, Schumann T, Schiefke I, Witzigmann H, Mossner J, Keim V. Decrease of CA 19-9 during chemotherapy with gemcitabine predicts survival time in patients with advanced pancreatic cancer. Br J Cancer. 2000; 82:1013-1016.

42. Stern P, Friedecky B, Bartos V, Bezdickova D, Vavrova J, Uhrova J, Rozprimova L, Zima T, Palicka V. Comparison of different immunoassays for CA 19-9. Clin Chem Lab Med. 2001; 39:1278-1282.

43. Lin DY, Wei LJ. The Robust Inference for the Cox Proportional Hazards Model. J Am Stat Assoc. 1989; 84:1074-1078. 